\title{
Perceived range, perceived velocity, and perceived duration of the body rotating in the frontal plane
}

\author{
Atsuki Higashiyama \\ Ritsumeikan University, Kyoto, Japan \\ AND \\ Kazuo Koga \\ Nagoya University, Nagoya, Japan
}

\begin{abstract}
We investigated perceived range, perceived velocity, and perceived duration of the body rotating in the frontal plane (in roll). Specifically, to examine how shear to the otoliths in the inner ears and tactile pressure to the trunk affect judgments of range and velocity, in two experiments, we manipulated rotating range $\left(30^{\circ}-160^{\circ}\right)$, rotating velocity $\left(1.8^{\circ} / \mathrm{sec}\right.$ to $\left.9.6^{\circ} / \mathrm{sec}\right)$, mean tilt of the body $\left(-60^{\circ}, 0^{\circ}\right.$, and $\left.60^{\circ}\right)$, and exposure to the visual vertical. Thirty-three normal or blindfolded participants made verbal judgments of range, velocity, and duration for each combination of these factors. The exponents of the power functions fitted to these judgments were, as a first approximation, .94, .61 , and .84 for range, velocity, and duration, respectively, and perceived velocity was proportional to the ratio of perceived range to perceived duration $(r=.91)$. These results suggest that the vestibular and somatosensory inputs are effective on judgments of range, but less so on judgments of velocity, and that perceived velocity may be determined as a ratio of perceived range to perceived duration. In addition, we found that (1) when the range the body has traveled is constant, the perceived range increases as the objective velocity decreases (proprioceptive $\tau$ effect); (2) self-motion through the tilted roll sometimes enlarges perceived range and perceived duration but reduces perceived velocity; and (3) the exposure to the visual vertical reduces variability of judgments for range and velocity and also reduces perceived range and perceived velocity of self-motion within a small range through the vertical roll.
\end{abstract}

When the head is tilted while the trunk is held vertical, gravity provides us with acceleration that stimulates the vestibular system in the inner ears. The gravitational acceleration is resolved into shear (a component acting tangentially on the otoliths in the utricles and saccules) and tension (a component acting at right angles on the otoliths). Shear, not tension, is the primary component that determines perceived tilt of the head (Benson, 1990; Benson, Spencer, \& Stott, 1986; Parker, Gulledge, Tubbs, \& Littlefield, 1978). However, it has been suggested that when the whole body, including the trunk, is progressively largely tilted, somatosensory inputs play an increasing role in the determination of body orientation (Lackner, 1992; Lackner \& Graybiel, 1979; Yardley, 1990). It is still debatable whether the critical contributor to body orientation comes from the vestibular or the somatosensory system (Bronstein, 1999; Howard, 1982; Lackner \& DiZio, 2005).

In this study, for the body passively rotated in roll (see Figure 1), we investigated perceived range, perceived velocity, and perceived duration. In this situation, the prevailing variables influencing perceived range are shear to the otoliths and pressure to the trunk. If these variables are registered at the starting and the goal orientations of the rotating body, the entire range the body has rotated is perceived as a difference of body orientations. Since accurate orientation judgments have been obtained for the body that is tilted $45^{\circ}$ or less from the gravitational vertical (Higashiyama \& Koga, 1998), it can be suggested that range judgments also will be accurate.

Perception of the duration that is needed for the body to move between different orientations may be basically determined by the inner clock - that is, by counting the number of unit durations that are implicitly ticked off while the body is moving (e.g., Treisman, Cook, Naish, \& MacCrone, 1994). The unit duration is assumed to be a physiological pacemaker such as heart beat or respiration rate. However, we do not perceive duration accurately, because the internal clock is disturbed by body temperature (e.g., Lockhart, 1967), features of the presented visual stimuli (e.g., Long \& Beaton, 1980; Ono \& Kawahara, 2007), and the participants' interest or attention (e.g., S. W. Brown, 1997; Hicks, Miller, Gaes, \& Bierman, 1977; Hicks, Miller, \& Kinsbourne, 1976). Despite this complexity of the temporal system, Eisler (1976), reviewing many studies concerning time perception, suggested that perceived duration generally grows less rapidly than the objective duration.

A. Higashiyama, achan@It.ritsumei.ac.jp 
If the range between body orientations is correctly perceived by shear to the otholiths and pressure to the trunk, and if the duration needed to move between them is perceived by the inner clock, we can formally determine perceived velocity of self-motion, $V^{\prime}$, by a ratio of perceived range, $R^{\prime}$, to perceived duration, $T^{\prime}$ (i.e., $V^{\prime}=R^{\prime} / T^{\prime}$ ), or by registering a small range over unit duration (i.e., $V^{\prime}=\Delta R / \Delta T$ ). The first formula predicts that perceived velocity is not accurate because perceived duration is not accurate, whereas the second formula predicts that perceived velocity is accurate because unit duration is constant (i.e., the heartbeat or respiration rate is assumed not to change while the body is moving). Since there are very few studies that have ever examined perceived velocity of self-motion (Higashiyama \& Koga, 2002; Parker, Wood, Gulledge, \& Goodrich, 1979), it is worth asking how the perceived velocity of self-motion is related to perceived range and perceived duration.

To examine other functional aspects of shear to the otoliths or pressure to the trunk, we controlled the mean tilt of the body when it rotated from one orientation to another. For symmetric self-motion through a vertical roll, either shear to the otoliths or pressure to the trunk is equivalent on the left and the right sides of the body, but for selfmotion through a nonvertical roll, it is larger on one side of the body than that on the other. As a result, tactile pressure and muscular tension in the body are progressively enhanced as the mean tilt of the body increases. However, the utricular maculae are less sensitive as the head is tilted, because they are most sensitive when the head is upright and become less so when the head is inclined (LechnerSteinleitner, 1978; Young, Oman, \& Dichgans, 1975).

Another variable examined in this study was visual inputs that suggested the direction of gravity. We predicted that information about the visual vertical would be useful for registering body orientation accurately and improving judgments of range and velocity. There are a large number of studies concerning cross-modal interaction between vision and postural orientation. Some studies have demonstrated that a stationary (Lackner \& Graybiel, 1983; Nemire \& Cohen, 1993; Witkin, 1949) or a dynamic (Allison, Howard, \& Zacher, 1999; Dichgan, Held, Young, \& Brandt, 1972; Higashiyama \& Koga, in press; Reason, Mayes, \& Dewhurst, 1982) visual pattern alters an observer's postural orientation, and other studies have demonstrated that the postural orientation affects the visual vertical, known as the A- and E-effects, respectively (see Bronstein, 1999, and Yardley, 1990, for patients losing vestibular or somatosensory functions, and see van Beuzekom \& van Gisbergen, 2000, and van Beuzekom, Medendorp, \& van Gisbergen, 2001, for normal adults). However, in very few studies have attempts been made to clarify how the visual vertical alters the perceived range and perceived velocity of self-motion.

\section{EXPERIMENT 1}

\section{Method}

Participants. Thirteen undergraduates ( 5 men and 8 women) served as the participants; none of them had any known defi- cits in motor or vestibular functions. They were paid for their participation.

Apparatus. The apparatus was a chair that was capable of rotating a participant in roll. The position of the chair was controlled by the analog voltage servo regulation system with an accuracy of $1^{\circ}$. The pivot on which the body rotated was located approximately at the midpoint between the eyes. A padded, adjustable iron structure was used to minimize shifts in the trunk, shoulder, and thigh. The head was stabilized with the restraining structure, so that the participant could not shake, nod, or incline his or her head. When being stabilized on the chair, the participant could not see parts of his or her body. To maintain verbal communication with the experimenter, the participant did not bite a board. The chair could be rotated clockwise $(\mathrm{CW})$ or counterclockwise (CCW). We will use a plus sign $(+)$ to signify $\mathrm{CW}$ from the gravitational vertical, and a minus sign (-) to signify $\mathrm{CCW}$ from the gravitational vertical. The possible maximal velocity of the chair was $12 \% \mathrm{sec}$, or $2 \mathrm{rpm}$. The participant faced a white hemisphere screen at a distance of about $180 \mathrm{~cm}$ from the midpoint of the eyes. The visual angle of the screen was $220^{\circ}$ in all directions, and its luminance was $1.9 \mathrm{~cd} / \mathrm{m}^{2}$.

The chair began to rotate from a starting position with a constant acceleration, reached a constant velocity $\left(1.8^{\circ}, 3.6^{\circ}\right.$, or $\left.7.2^{\circ} / \mathrm{sec}\right)$ within $420 \mathrm{msec}$, continued to rotate at this velocity for a preset duration, and returned to the goal position with a constant deceleration. For example, the angular acceleration and its duration that were needed for the chair to reach the $7.2^{\circ} / \mathrm{sec}$ velocity were $24^{\circ} / \mathrm{sec}^{2}$ and $420 \mathrm{msec}$, respectively. This order of angular acceleration (or deceleration) duration does not seem to have been enough to activate the semicircular canals and affect perceived velocity of self-motion. It is well documented that an acceleration duration of $5 \mathrm{sec}$ or more is needed to judge whether the body is rotating left or right (B. Clark, 1967; B. Clark \& Stewart, 1968a) or to verbally judge how fast the body is rotating (J. H. Brown, 1966; B. Clark \& Stewart, 1968b). More important, since the semicircular canals respond to angular acceleration of the body, they were not active while the body rotated at a constant velocity, which lasted after the impulsive acceleration.

Design. We used a factorial within-subjects design of roll (3) $\times$ range (3) $\times$ velocity (3) $\times$ direction (2). The roll, with respect to which the chair symmetrically moved, was $-60^{\circ}, 0^{\circ}$, or $60^{\circ}$. For the $60^{\circ}$ roll, for example, the chair moved between $60^{\circ}-\alpha$ and $60^{\circ}+\alpha$, where $\alpha$ is half the range that the chair rotated. Note that if the chair moved from the starting position to the goal position through the vertical roll, the shear was put equally on the left and right sides of the otoliths and the pressure was also put equally on either side of the body (e.g., the rotation from A to B in Figure 1), whereas when the body rotated through the nonvertical roll, the shear or pressure was put predominantly on the left side for the $-60^{\circ}$ roll or on the right side for the $60^{\circ}$ roll (e.g., the rotation from $\mathrm{C}$ to $\mathrm{D}$ ). In this experiment, the rotating range, $2 \alpha$, was $40^{\circ}, 60^{\circ}$, or $80^{\circ}$. The angular velocity of the chair was $1.8^{\circ}, 3.6^{\circ}$, or $7.2^{\circ} / \mathrm{sec}$. The rotating direction of the chair was $\mathrm{CW}$ or CCW. Each participant thus judged 54 combinations of these factors.

Procedure. Each participant sat blindfold on the chair, and his or her head, trunk, shoulders, and thighs were stabilized with the iron structure. On a given trial, the chair began to move from one preset position and stopped at another preset position. Immediately after the chair had stopped, the participant was required to judge the range that the chair had traveled, the velocity at which the chair had rotated, and the duration it had taken to travel the range.

For judgments of range, the participant used the unit of degrees. To make the participant's judgments easy, the experimenter, in advance, showed a watch to the participant and told him or her that if his or her body rotated like the hour hand and stayed at the position, say, of 1 o'clock, it would correspond to a $30^{\circ}$ tilt from the gravitational vertical. Perceived velocity of self-motion was judged relative to velocity of the second hand on a clock. The experimenter again showed the watch to the participant and asked him or her to designate the velocity of the second hand " 10 " (note that a second hand moves at the velocity of $6^{\circ} / \mathrm{sec}$, or $1 \mathrm{rpm}$ ). For judgments of duration, the partici- 


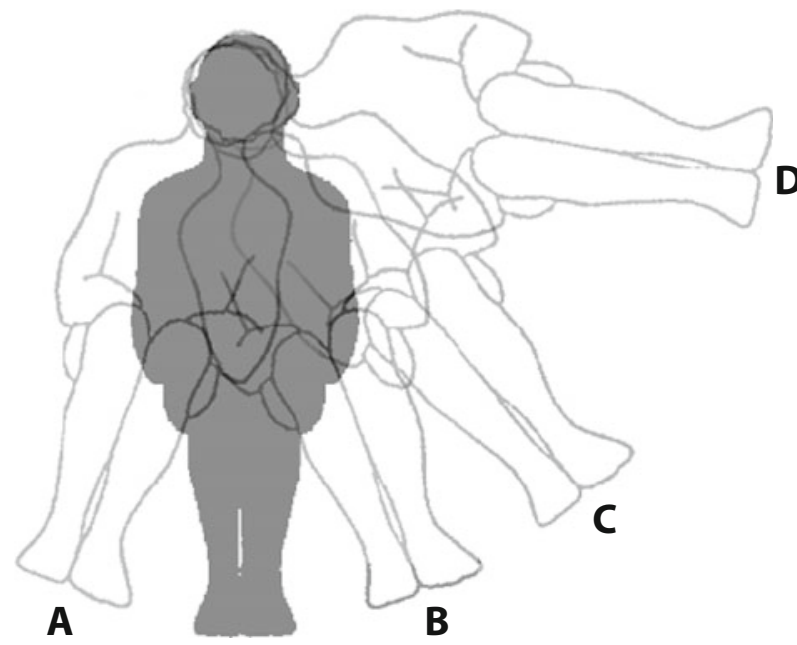

Figure 1. Upright body (filled body) and the body tilted $-20^{\circ}(\mathrm{A}), 20^{\circ}(\mathrm{B}), 40^{\circ}(\mathrm{C})$, and $80^{\circ}(\mathrm{D})$ in roll (outlined body). If the body rotates from $A$ to $B$, it travels the $40^{\circ}$ range clockwise through the vertical roll. If the body rotates from $C$ to $D$, it travels the $40^{\circ}$ range clockwise through the $60^{\circ}$ roll. Note that left/right or clockwise/counterclockwise is determined by the participant in the chair, not by the reader looking at the figure.

pant used the unit of seconds. The order in which range, velocity, and duration were judged was counterbalanced among the participants.

For each range-velocity combination, two orders of roll were run to save time in moving the chair to the starting position and to relieve the participant from the stabilized posture as soon as possible. In one order (i.e., $\mathrm{CW}$ successive trials), after the $-60^{\circ}$ roll, the $0^{\circ}$ roll was run before the $60^{\circ}$ roll. For example, for the $40^{\circ}-3.6^{\circ} / \mathrm{sec}$ combination under the $\mathrm{CW}$ successive trials, the chair first rotated from $-80^{\circ}$ to $-40^{\circ}$ through the $-60^{\circ}$ roll, then from -20 to $20^{\circ}$ through the $0^{\circ}$ roll, and finally from $40^{\circ}$ to $80^{\circ}$ through the $60^{\circ}$ roll, with a $3.6^{\circ} / \mathrm{sec}$ velocity for all the trials. In another order (i.e., CCW successive trials), the reverse was run. Judgments were alternated between the $\mathrm{CW}$ successive trials for one range-velocity combination and the $\mathrm{CCW}$ successive trials for the other range-velocity combination. The $\mathrm{CW}$ and the $\mathrm{CCW}$ successive trials for each combination were separated by those for other combinations. Each participant followed either of two different orders of the range-velocity combinations, which were randomly determined. Each participant completed all judgments in an hour, taking about 1 min per trial.

The $\mathrm{CW}$ and the $\mathrm{CCW}$ successive trials were useful for reducing possible postural aftereffects - the sense of the position of a limb, the head, or the eye, which may be temporarily affected when an asymmetrical posture is maintained for some time (F. J. Clark \& Horch, 1986). On the CW successive trials, the roll to the left was followed by the roll to the right after the upright roll, so that the aftereffects of the pressure to the left side of the body, if any, were nullified by the subsequent pressure to its right side. On the CCW successive trials as well, the aftereffects of the pressure to the right side were canceled out by the subsequent pressure to its left side.

\section{Results}

The judgments of range, velocity, and duration were analyzed separately. All the judgments were converted into logarithmic scores, and these scores were used for obtaining a geometric mean for each combination. A four-way (roll $\times$ velocity $X$ range $X$ direction) repeated measures ANOVA was performed on the scores for velocity, range, or duration.

Figure 2 shows mean perceived range as a function of roll tilt of the body. The left, center, and right panels are for the $40^{\circ}, 60^{\circ}$, and $80^{\circ}$ ranges, respectively, with the velocity as the parameter. The vertical bar attached to each mean in Figure 2 and the subsequent figures indicates an inverse transformation of the standard deviation based on logarithmic scores.

The mean perceived range increased as the objective range increased $[F(2,24)=208.5, p<.001]$. The mean judgments for the $40^{\circ}, 60^{\circ}$, and $80^{\circ}$ ranges were 39,57 , and 80 , respectively.

The mean perceived range decreased as the objective velocity increased $[F(2,24)=3.4, p<.05]$. The mean judgments for the $1.8^{\circ}, 3.6^{\circ}$, and $7.2^{\circ} / \mathrm{sec}$ velocities were 60,56 , and 51 , respectively.
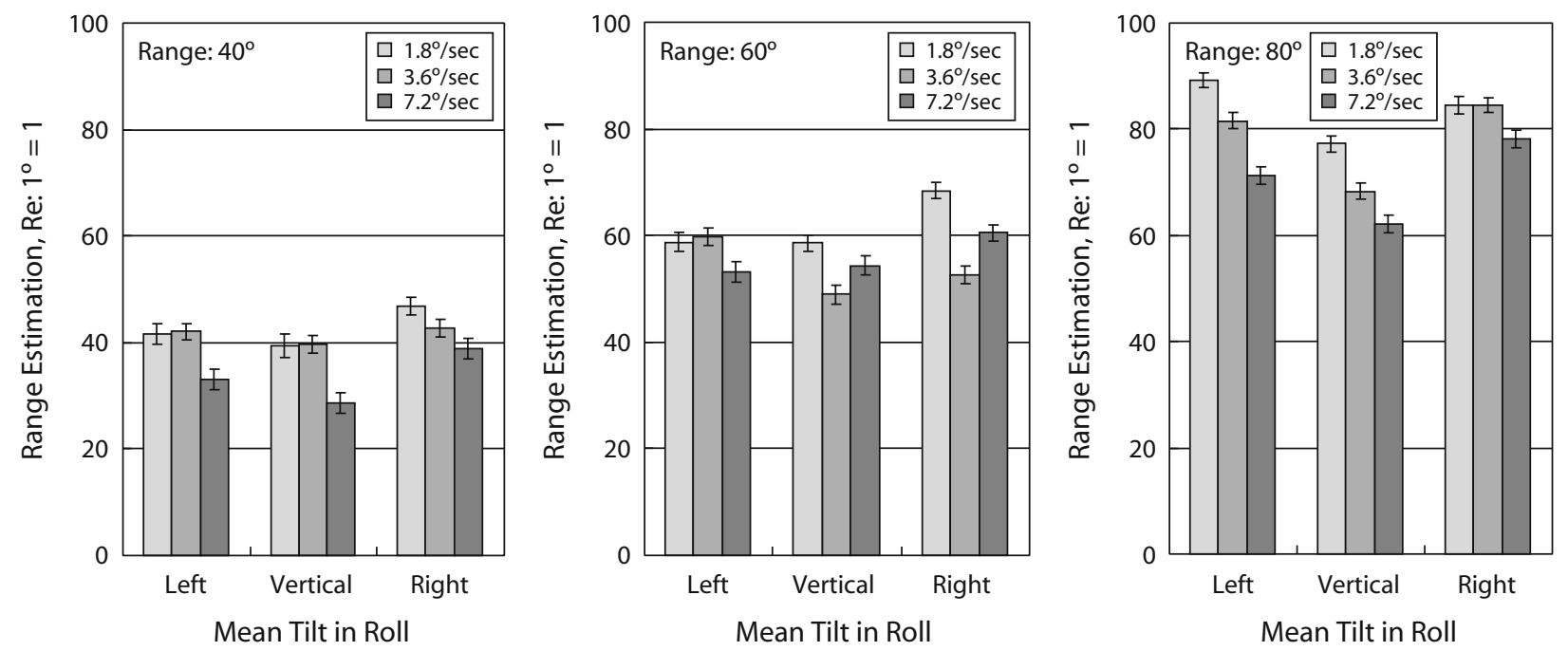

Figure 2. Mean range judgments as a function of mean roll tilt of the body. The left, center, and right panels show the results for the $40^{\circ}, 60^{\circ}$, and $80^{\circ}$ ranges, respectively. The parameter in each panel is velocity. 
The mean perceived range for the tilted rolls was larger than that for the vertical roll $[F(2,12)=4.9, p<.05]$. The mean judgments for the $-60^{\circ}, 0^{\circ}$, and $60^{\circ}$ rolls were 56 , 51 , and 60 , respectively.

The roll $\times$ range $\times$ direction interaction was significant $[F(4,48)=3.2, p<.05]$, indicating that the enlargement of perceived range for the tilted roll was limited to the specific range-direction combinations [for the $40^{\circ}-\mathrm{CCW}$ combination, $F(2,144)=5.4, p<.01$; for the $60^{\circ}-\mathrm{CCW}$ combination, $F(2,144)=3.8, p<.05$; and for the $80^{\circ}-$ CW combination, $F(2,144)=5.5, p<.01]$.

Figure 3 shows the mean perceived velocity as a function of roll tilt of the body. The left, center, and right panels are for the $1.8^{\circ}, 3.6^{\circ}$, and $7.2^{\circ} / \mathrm{sec}$ velocities, respectively, with the range as the parameter. Accurate judgments would be 3 for the $1.8 \% \mathrm{sec}$ velocity, 6 for the $3.6^{\circ} / \mathrm{sec}$ velocity, and 12 for the $7.2^{\circ} / \mathrm{sec}$ velocity.

The mean perceived velocity increased as the objective velocity increased $[F(2,24)=120.2, p<.001]$, and the mean perceived velocity was larger than the accurate judgments, as is shown in Figure 3. It is also noted that the mean perceived velocity grew less rapidly than the objective velocity: The mean judgments for the $1.8^{\circ}, 3.6^{\circ}$, and $7.2^{\circ} / \mathrm{sec}$ velocities were $6.2,9.7$, and 14.6 , respectively.

The main effect of roll was significant $[F(2,24)=4.0$, $p<.05]$. The mean perceived velocities for the $-60^{\circ}, 0^{\circ}$, and $60^{\circ}$ rolls were 9.2, 10.0, and 9.5, respectively, suggesting that the mean perceived velocity for the vertical roll was larger than that for the tilted roll. However, this tendency was significant for the $\mathrm{CW}$ rotation $[F(2,48)=$ $11.0, p<.001]$, but not for the CCW rotation.

The roll $\times$ velocity interaction was significant $[F(4,48)=4.5, p<.001]$. For the $1.8^{\circ} / \mathrm{sec}$ velocity, the mean perceived velocity for the $0^{\circ}$ roll was larger than that for the $-60^{\circ}$ or the $60^{\circ}$ roll $[F(2,72)=9.6, p<.001]$. For the $3.6 \%$ sec velocity, the difference in perceived velocity among rolls was not significant; for the $7.2^{\circ} / \mathrm{sec}$ velocity, the mean perceived velocity for the $60^{\circ}$ roll was larger than that for the $-60^{\circ}$ roll $[F(2,72)=3.1, p<.05]$. It is difficult to interpret this interaction in a meaningful way.

Figure 4 shows the mean perceived duration as a function of objective duration. For convenience, the objective range and objective velocity are attached to each objective duration on the abscissa. The parameter is the roll tilt. It was found that the mean perceived duration increased as the objective range increased $[F(2,24)=268.5, p<.001]$; the mean perceived duration also increased as the objective velocity decreased $[F(2,24)=256.9, p<.001]$, but the velocity $\times$ range interaction was not significant. Thus, the mean perceived duration increased as the objective duration increased, although it was underestimated, as is shown in Figure 4.

The main effect of roll was significant $[F(2,24)=4.3$, $p<.05$ ], suggesting that the mean perceived duration was generally larger for the tilted roll than for the vertical roll. The roll $\times$ velocity interaction was significant $[F(4,48)=$ $4.5, p<.01]$ : The simple main effect of roll was significant for the $1.8^{\circ} / \mathrm{sec}$ velocity $[F(2,72)=5.6, p<.01]$ and for the $3.6^{\circ} / \mathrm{sec}$ velocity $[F(2,72)=6.4, p<.001]$. The roll $\times$ range interaction was also significant $[F(4.48)=4.7, p<$ $.001]$ : The simple main effect of roll was significant for the $40^{\circ}$ range $[F(2,72)=12.1, p<.001]$. Taken together, these interactions suggest that the enlargement of perceived duration for tilted roll was likely to occur for the smallest duration and for the duration of $11.1 \mathrm{sec}$ or more.

\section{Discussion}

The mean perceived velocity, perceived range, and perceived duration increased as the objective velocity, objective range, and objective duration increased, respectively. Specifically, range was judged accurately, but perceived velocity was probably a decelerated function of objective velocity, and perceived duration was also a decelerated function of objective duration. Thus, the perceptual qualities arising from passive self-motion generally correspond with the physical counterparts.
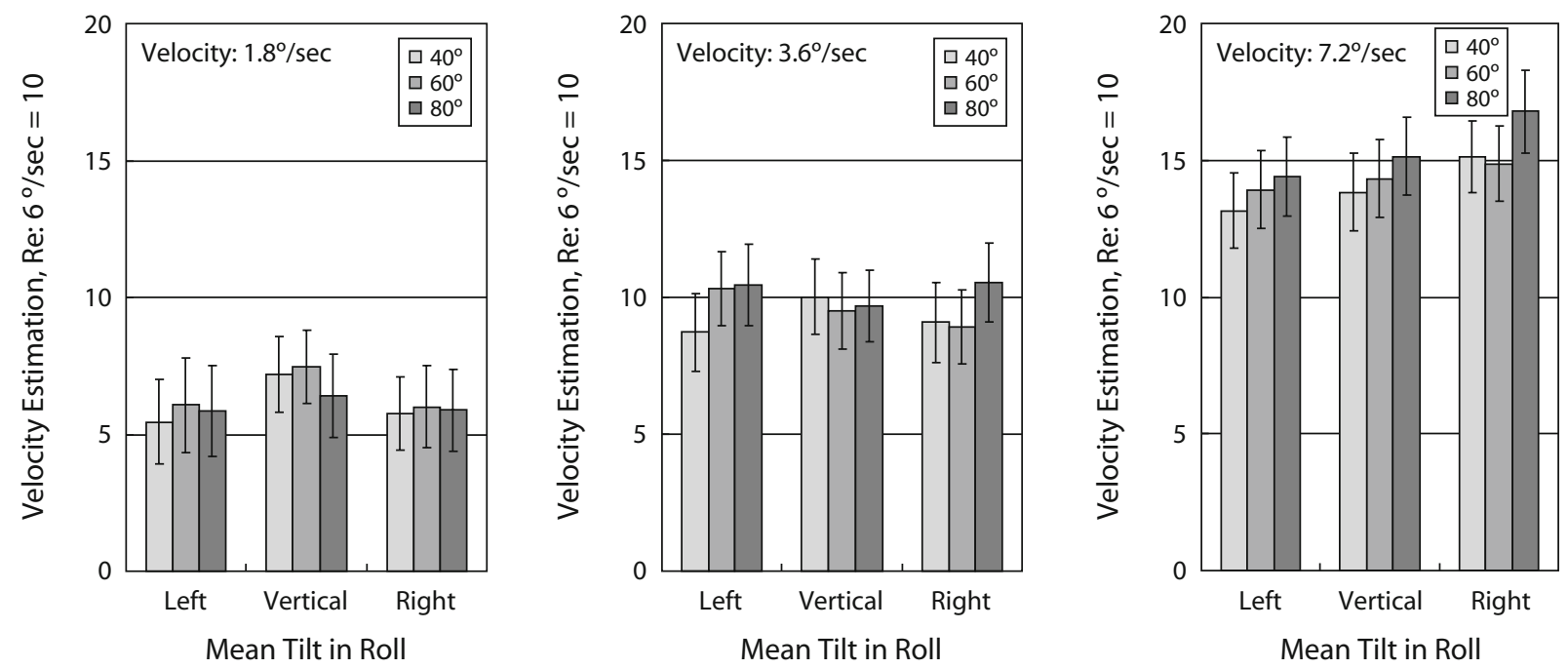

Figure 3. Mean velocity judgments as a function of mean roll tilt of the body. The left, center, and right panels show the results for the $1.8^{\circ}, 3.6^{\circ}$, and $7.2^{\circ}$ /sec velocities, respectively. The parameter in each panel is range. 


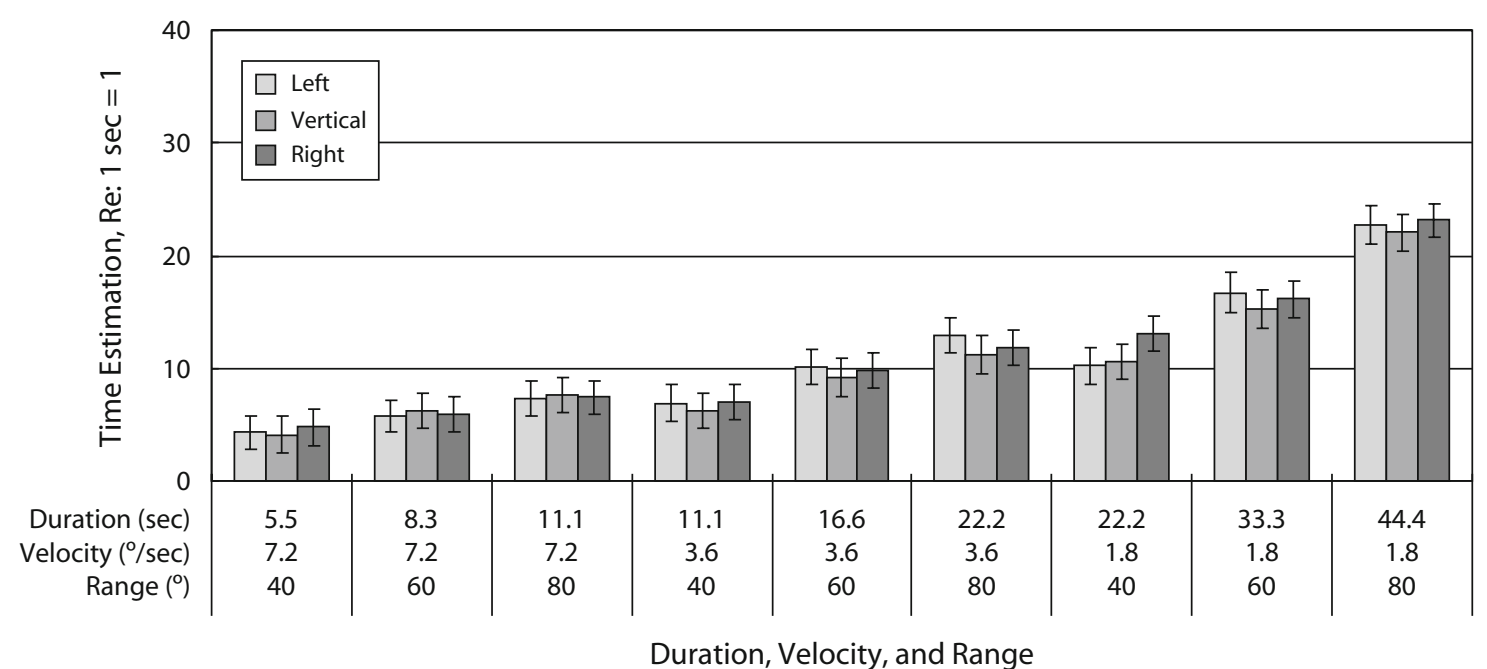

Figure 4. Mean duration judgments as a function of objective duration (also, of combination of range and velocity). The parameter is mean roll tilt of the body.

However, two findings disturbed this correspondence between perceptual quality and physical dimension. First, the mean perceived range decreased as the objective velocity increased. This may suggest that perceived range is directly affected by objective velocity, but another interpretation is possible: Perceived range increases with an increase in objective duration, since it takes much time for the slowly rotating body to arrive at a goal. Although we are not able to rule out either interpretation on the basis of the present data, we call this finding the proprioceptive $\tau$ effect, after the visual $\tau$ effect (Helson \& King, 1931), which means that the distance of apparent movement between two light points is perceived to enlarge as the interstimulus time interval increases.

The second pertains to the effects of roll tilt. We found that the mean perceived range was larger for the tilted roll than for the vertical roll and the mean perceived duration was also larger for the tilted roll than for the vertical roll. These findings suggest that tactile or proprioceptive inputs affect perceived range and perceived duration. When the body was tilted together with the chair, the body was pushed down on the iron structure of the chair, causing strong pressure and, sometimes, pain. To protect the body against pressure, the participants contracted their muscles reflexively.

In this connection, we wonder whether the change of potential energy of the body affected perceived range and perceived velocity of self-motion. When the body was being tilted right, the $\mathrm{CW}$ rotation of the body lifted up the participant's feet (i.e., a low-to-high change in potential energy), but the CCW rotation of the body took them down (i.e., a high-to-low change in potential energy). When the body was being tilted left, on the other hand, the $\mathrm{CW}$ or the $\mathrm{CCW}$ rotation produced the reverse change in potential energy. If the pressure to the body differed between the low-to-high and the high-to-low changes in potential energy, the $\mathrm{CW}$ rotation through the $-60^{\circ}$ roll and the CCW rotation through the $60^{\circ}$ roll would have produced the same perceived range or perceived veloc- ity, which would have differed from perceived range or perceived velocity for the CW rotation through the $60^{\circ}$ roll and for the CCW rotation through the $-60^{\circ}$ roll. The judgments of range or velocity suggest that we were not sensitive to a change in potential energy of the body (i.e., absence of the roll $\times$ range interaction).

\section{EXPERIMENT 2}

As was stated in the introduction, the aim of Experiment 2 was to examine the effects of visual framework on perceived range, perceived velocity, and perceived duration of self-motion. The participants in one group were blindfolded as in Experiment 1, and the participants in another group looked at a photograph of an upright woman. By viewing the picture, the participants were expected to note the direction of the visual vertical, register the difference in orientation between the retinal image of the picture and the vertical meridian of the retina, and perceive their own body orientation more accurately. We compared judgments of range, velocity, and duration between the picture conditions.

The second aim was to ensure reproducibility of the results of Experiment 1. In Experiment 2, we increased objective range and objective velocity (and, as a consequence, objective duration as well) by lifting the participant's feet above the head. Although judgments of range were accurate in Experiment 1, it was questionable whether the judgments would still be accurate for a larger range, because we have little experience in which the body is rotated and turns out upside down. However, if registration of the vestibular and somatosensory inputs at the starting and goal positions was accurate, the size of the range would not affect the perceived range. We can also suggest that if the outcomes of Experiment 1 were reproduced in Experiment 2, the method used here is reliable, and the data from the two experiments can be combined to construct scales for range, velocity, and duration. 


\section{Method}

Participants. Twenty undergraduates (11 men and 9 women) were newly recruited as participants; none of them had any known deficits in visual, motor, or vestibular functions. They were paid for their participation.

Apparatus. The apparatus was the same as that used in Experiment 1 . We took a chromatic photograph of a woman $(157 \mathrm{~cm}$ tall, $27 \mathrm{~cm}$ wide at the head, and $47 \mathrm{~cm}$ wide at the trunk) and mounted it on plywood that was cut out into the woman's figure, with a support at the rear of the picture. This picture was presented in front of the hemisphere screen and was $150 \mathrm{~cm}$ away from the participant's eyes.

Design. We used a factorial mixed design of picture $(2) \times$ roll (3) $\times$ range $(2) \times$ velocity $(2) \times$ direction $(2)$. Picture was a betweensubjects variable: Ten participants were required to look at the picture throughout the making of judgments (exposure condition), and the other 10 participants were blindfolded throughout the making of judgments (occlusion condition). Each participant judged 24 combinations of roll $\times$ range $\times$ velocity $\times$ direction. The roll, with respect to which the chair moved symmetrically, was $-60^{\circ}, 0^{\circ}$, or $60^{\circ}$. The rotating range of the chair was $80^{\circ}$ or $160^{\circ}$; the rotating velocity was $2.4 \% \mathrm{sec}$ or $9.6^{\circ} / \mathrm{sec}$; the rotating direction was $\mathrm{CW}$ or $\mathrm{CCW}$.

Procedure. Each participant generally followed the same procedure as that in Experiment 1. The participant's head, trunk, shoulders, and thighs were stabilized with the iron structure. The participants in the exposure condition were told that the woman stood erect and her orientation might be available when range, velocity, and duration of self-motion were judged. The participants in the occlusion condition were told that despite the eyes' being occluded, they could judge range, velocity, and duration of self-motion by the sense of the body.

On a given trial, the chair began to move from one preset position and stopped at another preset position. Immediately after the chair had stopped, in the same way as in Experiment 1, the participant was required to judge the range that the chair had traveled, the velocity at which the chair had rotated, and the duration that had been needed to rotate through the range. The order in which the range, velocity, and duration were judged was counterbalanced among the participants. For each range-velocity combination, the $\mathrm{CW}$ and the $\mathrm{CCW}$ successive trials were run as in Experiment 1. The participants followed either of two orders of the range-velocity combinations, which were randomly determined.

\section{Results}

The judgments of range, velocity, and duration were analyzed separately. All the judgments were converted into logarithmic scores, and these scores were used for obtaining a geometric mean for each combination. A four-way ANOVA, with picture as a between-subjects variable and roll, velocity, and range as within-subjects variables, was performed on these scores for velocity, range, or duration. Direction was collapsed by averaging the scores for the $\mathrm{CW}$ and $\mathrm{CCW}$ trials, because a five-way ANOVA might make it difficult to interpret the results and direction had been found to be minor in Experiment 1.

Figure 5 shows the mean perceived range as a function of roll tilt of the body. The left and right panels are for the $80^{\circ}$ and $160^{\circ}$ ranges, respectively, with the picture and velocity as the parameters. The mean perceived range increased with an increase in objective range $[F(1,18)=267.7, p<$ $.001]$. As can be seen in Figure 5, when the objective range changed from $80^{\circ}$ to $160^{\circ}$, the mean judgment changed from 67 to 146, and this change for judgments was almost the same as the change for objective ranges.

The mean perceived range increased with a decrease in objective velocity $[F(1,18)=26.0, p<.001]$. For the $2.4^{\circ}$ and $9.6^{\circ} / \mathrm{sec}$ velocities, the mean perceived ranges were 110 and 88 , respectively.

The picture $\times$ roll interaction was almost significant $[F(2,36)=3.2, p=.052]$. Under the exposure condi-
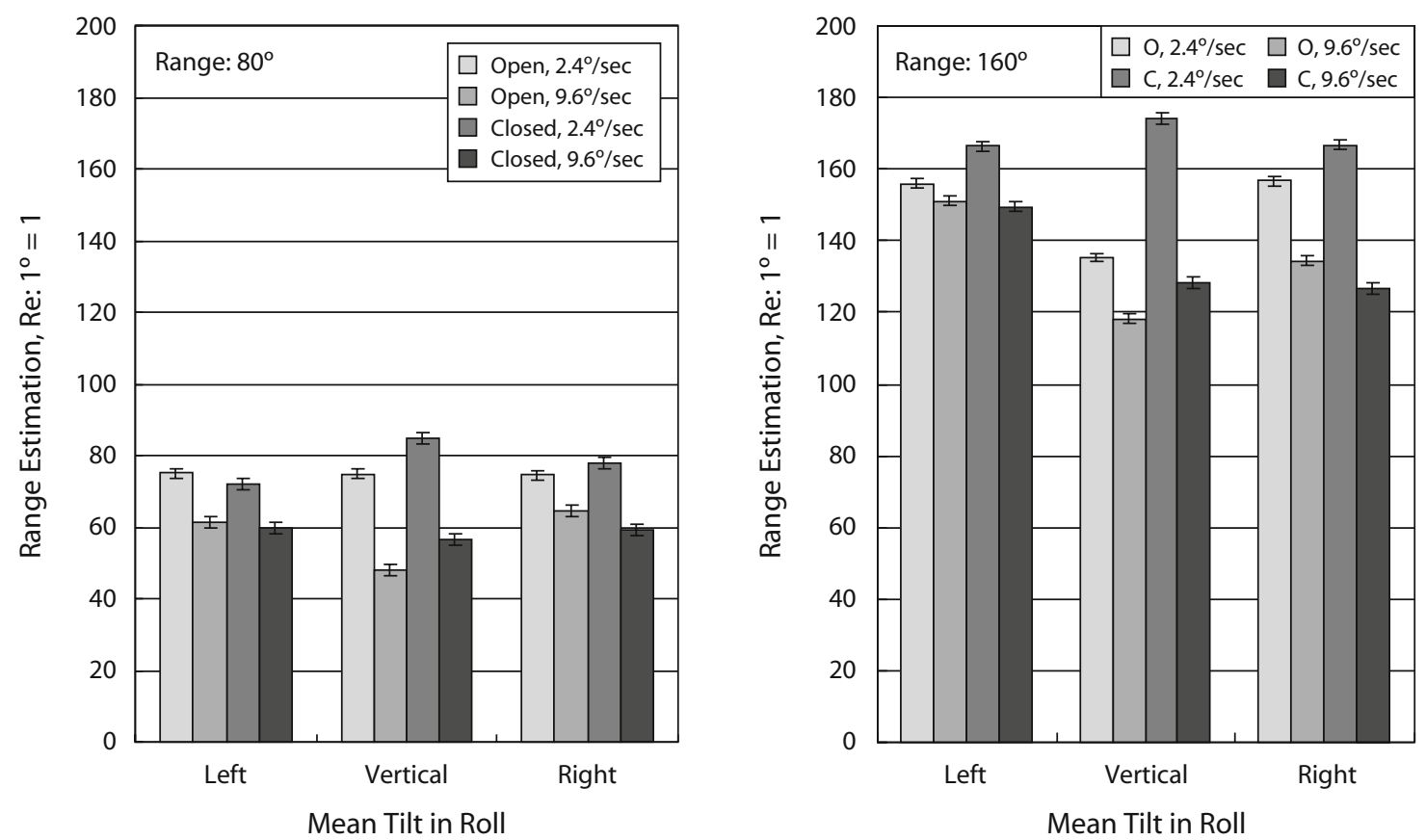

Figure 5. Mean range judgments as a function of mean roll tilt of the body. The left and right panels show the results for the $80^{\circ}$ and $160^{\circ}$ ranges, respectively. The parameters in each panel are picture (open and closed eyes) and velocity $\left(2.4^{\circ}\right.$ and $\left.9.6^{\circ} / \mathrm{sec}\right)$. 
tion, the mean perceived ranges were 102,87 , and 100 for $-60^{\circ}, 0^{\circ}$, and $60^{\circ}$, respectively, but under the occlusion condition, the mean perceived ranges were 102, 102, and 100 for the $-60^{\circ}, 0^{\circ}$, and $60^{\circ}$, respectively. Clearly, the mean perceived range was much reduced when the photograph was viewed with the vertical roll.

Figure 6 shows the mean perceived velocity as a function of roll tilt of the body. The left and right panels of Figure 6 are for the $2.4^{\circ}$ and $9.6^{\circ} / \mathrm{sec}$ velocities, respectively, with picture and range as the parameters. Accurate judgments would be 4 for the $2.4^{\circ} / \mathrm{sec}$ velocity and 16 for the $9.6^{\circ} \mathrm{sec}$ velocity. The mean perceived velocity increased with an increase in objective velocity $[F(1,18)=155.6$, $p<.001]$. However, when the objective velocity increased four times, the mean judgment did not increase so much; It was only 2.4 times as large, as is shown in Figure 6 (6.6 for the $2.4 \% \mathrm{sec}$ velocity and 16.1 for the $9.6^{\circ} / \mathrm{sec}$ velocity).

The velocity $\times$ range interaction was significant $[F(1,18)=6.8, p<.05]$. This interaction indicates that for the $2.4 \%$ sec velocity, the mean perceived velocity for the $80^{\circ}$ range was not significantly different from that for the $160^{\circ}$ range, whereas for the $9.6^{\circ} / \mathrm{sec}$ velocity, the mean perceived velocity for the $80^{\circ}$ range was significantly smaller than that for the $160^{\circ}$ range $[F(1,36)=10.5, p<.001]$.

The roll $\times$ range interaction was significant $[F(2,36)=$ $4.3, p<.05$ ], indicating that for the $80^{\circ}$ range, the mean perceived velocity for the tilted roll was significantly smaller than that for the vertical roll $[F(2,72)=4.0, p<.05]$, whereas for the $160^{\circ}$ range, the mean perceived velocity was not significantly different among the roll conditions.

The roll $\times$ range interaction also indicated that for the $0^{\circ}$ roll, the mean perceived velocity for the $80^{\circ}$ range was

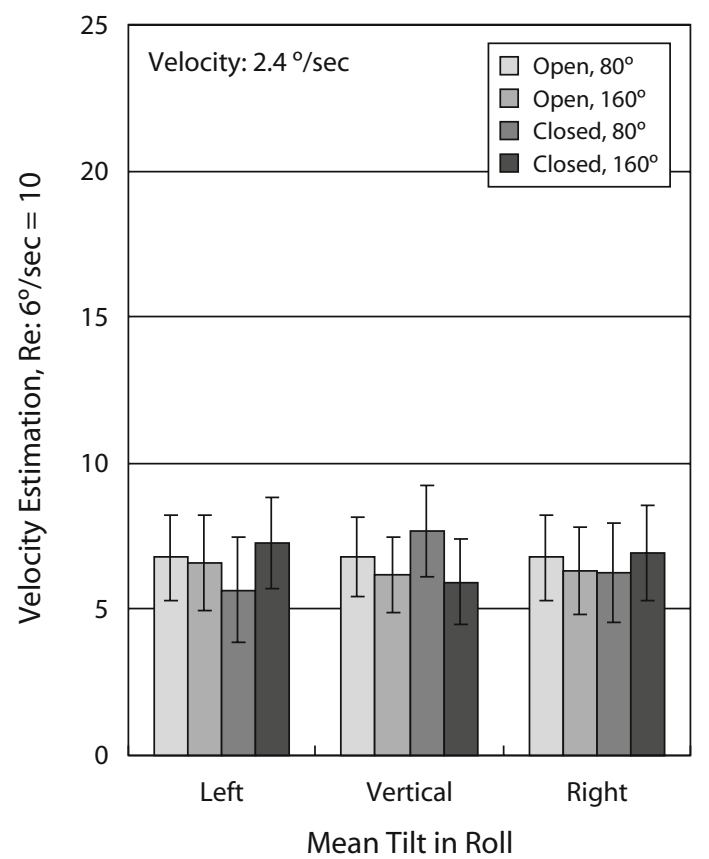

not significantly different from that for the $160^{\circ}$ range, whereas for the $-60^{\circ}$ roll $[F(1,54)=6.7, p<.05]$ and the $60^{\circ}$ roll $[F(1,54)=4.4, p<.05]$, the mean perceived velocity for the $80^{\circ}$ range was significantly smaller than that for the $160^{\circ}$ range.

The picture $\times$ roll $\times$ range interaction was significant $[F(2,36)=4.6, p<.05]$. This means that for the $80^{\circ}$ range, the mean difference in perceived velocity between the picture conditions was greater for the vertical roll (4.1) than for the $-60^{\circ}(2.1)$ and $60^{\circ}(0.6)$ rolls, but for the $160^{\circ}$ range, the difference was not significant $\left(2.3\right.$ for the $-60^{\circ}$ roll, 1.6 for the $0^{\circ}$ roll, and 2.5 for the $60^{\circ}$ roll).

Figure 7 shows the mean perceived duration as a function of objective duration. The parameters are picture and roll. It was found that the mean perceived duration increased with an increase in objective range $[F(1,18)=316.2, p<$ $.001]$; it also increased with a decrease in objective velocity $[F(1,18)=685.3, p<.001]$, but the velocity $\times$ range interaction was not significant. This implies that the mean perceived duration increased as the objective duration increased, although the perceived duration was likely to be underestimated, as is shown in Figure 7.

We compared variability of judgments between the exposure and the occlusion conditions. As a measure of variability, we used the standard deviation of the individual logarithmic scores for each combination of roll $\times$ range $X$ velocity in each picture condition. For judgments of range, the mean variability of the 12 combinations in the exposure condition (.135) was significantly different from that in the occlusion condition (.169) $[t(11)=3.47, p<.01]$. For judgments of velocity, the mean variability in the exposure condition (.144) was significantly different from

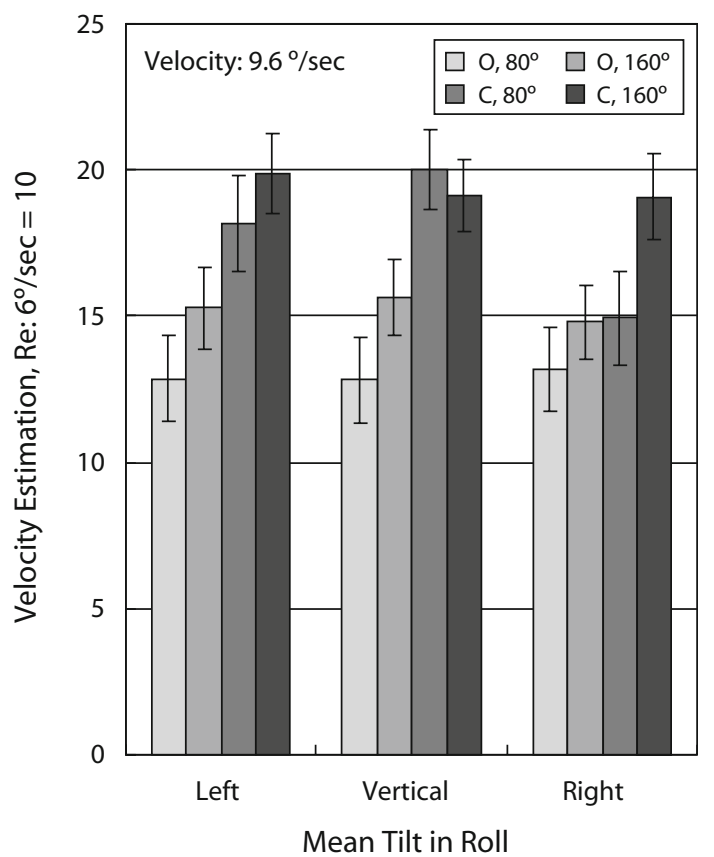

Figure 6. Mean velocity judgments as a function of mean roll tilt of the body. The left and right panels show the results for the $2.4^{\circ}$ and $9.6^{\circ} / \mathrm{sec}$ velocities, respectively. The parameters are picture (open and closed eyes) and range $\left(80^{\circ}\right.$ and $160^{\circ}$ ). 


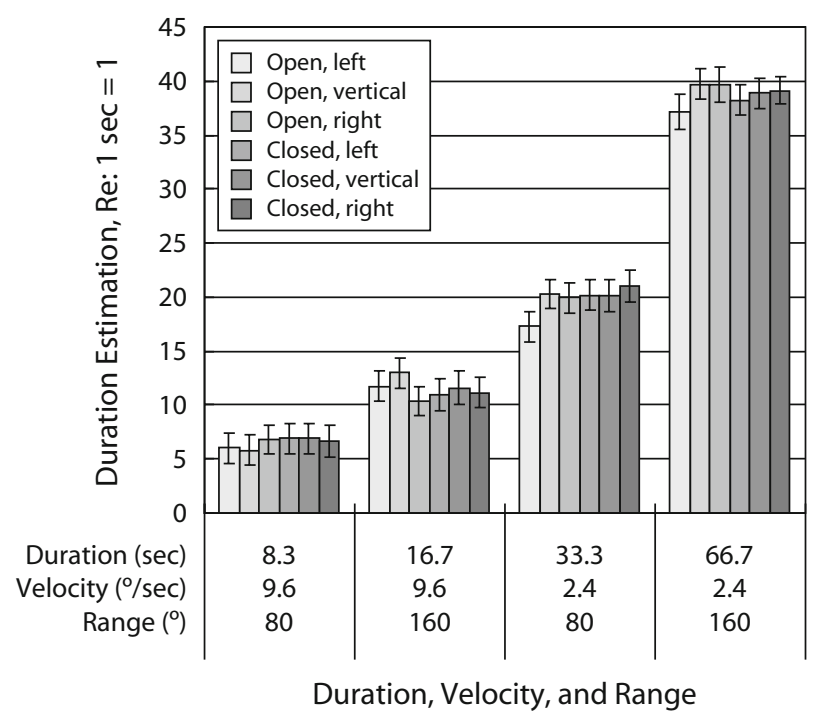

Figure 7. Mean duration judgments as a function of objective duration (also, of combination of range and velocity). The parameters are mean roll tilt (left, vertical, and right) and picture (open and closed eyes).

that in the occlusion condition $(.175)[t(11)=2.94, p<$ $.05]$. For judgments of duration, however, the difference was not significant between the exposure (.146) and the occlusion (.150) conditions. Thus, looking at the picture reduced variability of judgments for range and velocity, but not for duration.

\section{COMBINATION OF EXPERIMENTS 1 AND 2}

\section{Scales for Range, Velocity, and Duration}

Although it is clear that perceived range, perceived velocity, and perceived duration are complexly affected by a number of variables, it is interesting to construct, as a first approximation, psychophysical scales for range, velocity, and duration. Figure 8 again shows the mean judgments of range (top), velocity (middle), and duration (bottom). For each perceptual quality, the mean judgments in Experiments 1 and 2 were plotted on $\log -\log$ coordinates as a function of its physical counterpart. The diagonal line in each panel represents accurate judgments: In the case of range or duration, it is the line passing through the origin $(0,0)$, and in the case of velocity, it is the line passing through the point $(6,10)$, because we defined the $6^{\circ} / \mathrm{sec}$ velocity as 10 .

For the scale for range, we used the mean judgments for 9 (Experiment 1) or 12 (Experiment 2) combinations for each of the ranges we used in each experiment. We fitted a power function to these judgments by a least-square criterion. The resulting scale was

$$
R^{\prime}=1.12 R^{0.94},
$$

where $R^{\prime}$ is perceived range and $R$ is the objective range. The coefficient of determination, $r^{2}$, was .91 $(N=51)$. Equation 1 and the top of Figure 8 reveal that the results of
Experiment 1 are consistent with those of Experiment 2, and the perceived range is very accurate.

Similarly, we obtained the scale for velocity:

$$
V^{\prime}=4.14 V^{0.61} \text {, }
$$

where $V^{\prime}$ is perceived velocity and $V$ is the objective velocity. The value of $r^{2}$ was $.92(N=51)$. Equation 2 and the middle of Figure 8 reveal that the results of Experiments 1 and 2 are consistent, but the perceived velocity grows less rapidly than the objective velocity, and the judgments of perceived velocity, especially for small objective velocities, are larger than the accurate judgments.

We also obtained the scale for duration:

$$
T^{\prime}=.99 T^{0.84}
$$

where $T^{\prime}$ is perceived duration and $T$ is the objective duration. The value of $r^{2}$ was $.97(N=51)$. Equation 3 and the bottom of Figure 8 reveal that the results of Experiment 1 agree with those of Experiment 2, but the perceived duration grows less rapidly than the objective duration, and the judgments of perceived velocity are, overall, smaller than the accurate judgments.

One may ask about reliability of the method of absolute judgments we have used here. Despite the differences in participants and stimuli between Experiments 1 and 2, the results are similar to each other in each perceptual quality, and high degrees of goodness of fit for the power function $\left(r^{2}>.91\right)$ were obtained. We therefore maintain that the method of absolute judgments is reliable.

\section{Relation Among $R^{\prime}, V^{\prime}$, and $T^{\prime}$}

In physics, there is a simple and rigid relation among range, velocity, and duration. So, it is interesting to examine how the relation among $R^{\prime}, V^{\prime}$, and $T^{\prime}$ is similar to the physical relation. Specifically, as is showen in Figure 9, we examined whether perceived velocity is proportional to the ratio of perceived range to perceived duration: $V^{\prime}=$ $1.67 \times R^{\prime} / T^{\prime}$. The value of 1.67 (i.e., 10/6) in this equation is the scale factor, to adjust the relation among the units. The diagonal line represents perfect agreement between the obtained and the predicted velocities. Clearly, the data points scatter around the diagonal line, although the variation increases as the perceived velocity increases. The correlation between the obtained and the predicted velocities was .91 $(N=51)$ for all the data in both experiments. This high correlation seems to be attributable to small perceived velocities. The correlation for the velocities of 10 and less was $.85(N=26)$, and the correlation for the velocities over 10 was $.63(N=25)$. The simple relation among $R^{\prime}, V^{\prime}$, and $T^{\prime}$ probably holds only when a body rotates at a velocity of $6 \% \mathrm{sec}$ or less.

\section{GENERAL DISCUSSION}

\section{Basic Processing}

If we correctly registered the vestibular and somatosensory inputs and if the inner clock worked accurately, the exponents in Equations 1-3 that were fitted to the data would be equal to unity. The exponent of the scale for 

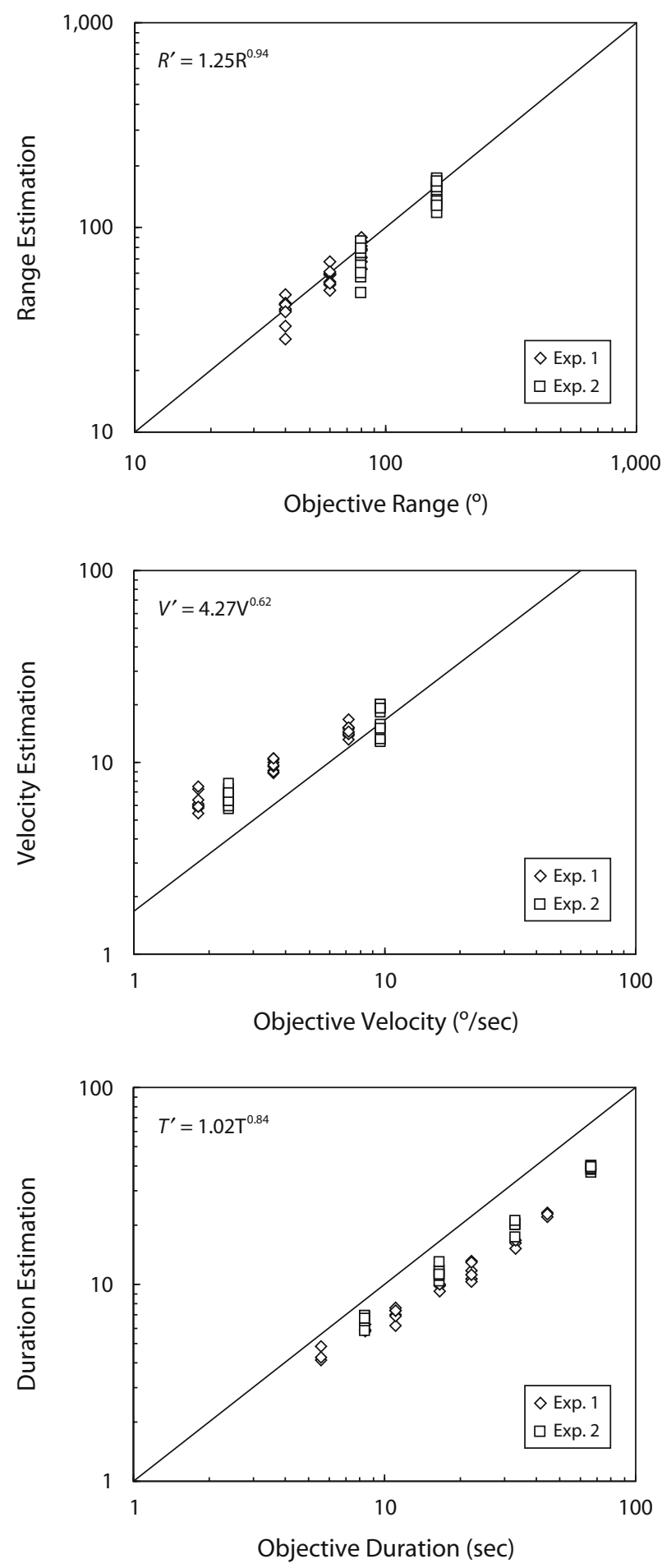

Figure 8. Psychophysical relations for range (top), velocity (middle), and duration (bottom) on log-log coordinates. The data points in each panel are the mean judgments in Experiments 1 and 2.

range, .94, confirms this assumption: We registered the vestibular and somatosensory inputs at the starting and goal orientations of self-motion and perceived the difference between them as perceived range. The exponent obtained here, however, is smaller than the exponent for self-motion during linear oscillation (Parker et al., 1979).

The exponent of the scale for velocity (.61) suggests that the vestibular and somatosensory inputs were not very effective in judging velocity: Doubling objective velocity increased perceived velocity to only 1.53 times as large. This low exponent was not improved even when the participants were exposed to the visual vertical to enhance accuracy of body orientation. This suggests that although we perceive the range of self-motion accurately, we do not judge a small range with respect to unit duration (i.e., $\Delta R / \Delta T)$.

The exponent of the scale for duration (.84) implies that perceived duration is compressive, but this value is consistent with the results of previous studies that have shown that the exponent of the power function for duration approximates .9 as an average of numerous conditions (Eisler, 1976). If we perceive velocity as the combination of compressive perceived duration with accurate perceived range, the scale for velocity is also compressed, with the exponent less than unity. This explanation is indeed supported by Figure 9, in which the mean perceived velocity is approximately proportional to the ratio of perceived range to perceived duration.

The basic processing suggested by the present results regarding $R^{\prime}, V^{\prime}$, and $T^{\prime}$ can be summarized as follows.

1 . Perceived range is accurately determined by the vestibular and somatosensory inputs as a difference in body orientation.

2. Perceived duration is probably determined by the inner clock, and it has a feature of a power function with an exponent smaller than unity.

3 . Perceived velocity is determined by the ratio of perceived range to perceived duration. As a result, the scale for velocity is also a power function with negative acceleration.

This basic processing model has two implications. First, the primary function of the vestibular and somatosensory organs that are activated by linear acceleration, including gravity, is the perception of body orientation and selfmotion range, rather than the perception of self-motion velocity (Howard, 1982, p. 360). The second is related to the effect of the visual vertical. Rachlin (1966) obtained an exponent of the power function for visual velocity (.75) for a luminous spot moving linearly across the frontoparallel screen. Algom and Cohen-Raz (1984) also obtained an exponent of the power function for visual velocity (.63) by observation of a luminous spot moving on a circular track on the screen. These suggest that we are insensitive to visual velocity, so that even if we are exposed to visual stimuli while the body rotates, the scale for vestibulotactile velocity is not improved drastically.

\section{Proprioceptive $\tau$ Effect}

The basic processing of self-motion is often contaminated by several biological and psychological factors. One is the proprioceptive $\tau$ effect: If the range the body has traveled in roll is constant, the mean perceived range increases as the objective velocity decreases. In other words, the mean perceived range increases with an increase in the 


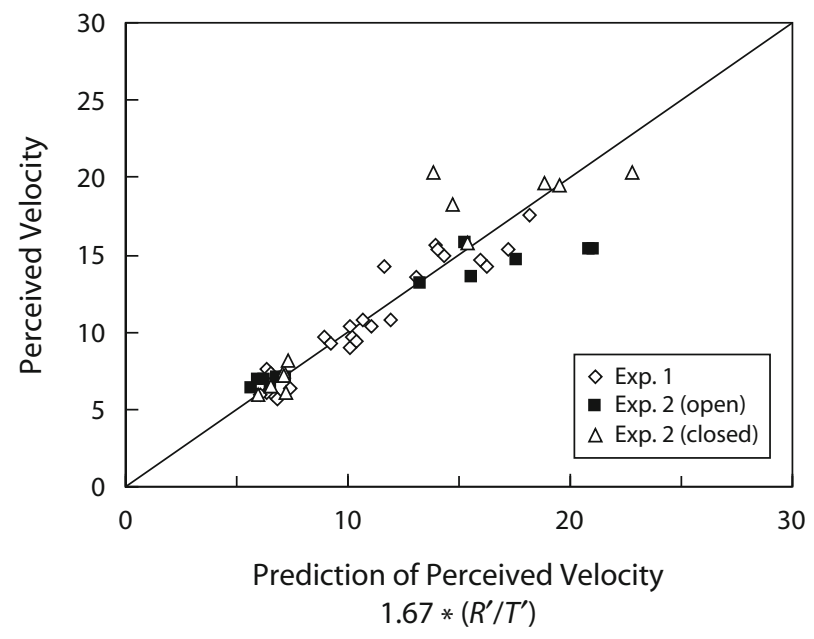

Figure 9. Mean perceived velocity obtained as a function of perceived velocity predicted by $1.67 \times R^{\prime} / T^{\prime}$. The data points in each panel are the mean judgments in Experiments 1 and 2.

duration needed to travel the range. Whichever is relevant, it is certain that the proprioceptive $\tau$ effect is induced by a variable pertinent to time.

The proprioceptive $\tau$ effect was intensive, because it was detected in both experiments of this study. The proprioceptive $\tau$ effect is also extensive: The effect has been found not only for passive self-motion, as in this study, but also for active self-motion. Hollins and Goble (1988) required participants to slide their index finger laterally along a straight path and to estimate the distance through which the finger moved. The magnitude estimates of distance were then found to decrease as finger velocity increased.

It is difficult to explain the proprioceptive $\tau$ effect in terms of aftereffects of the semicircular canals. The endolymph liquid of the ampullae in the canals is understood in terms of three phases of head motion. First, when the head starts rotating, the endolymph liquid of the ampulla lags behind, bending the cupula in the direction opposite to that of the head rotation. Second, when the head rotates for a while at a constant angular velocity, the cupula returns to its resting position, so that no signal is discharged from the canals. Third, when the head rotation is decelerated to a stop, the cupula displaces in the same direction as that in which the head has rotated, and it takes up to about $30 \mathrm{sec}$ before the behavioral effects of this defection, like experiences of rotating sensation and nausea, are dissipated (Howard, 1982, p. 347). If the aftereffects arising in the last phase had intruded into the judgments of angular range in this study, perceived range of the body rotation would have increased as its objective velocity increased.

It seems that the proprioceptive $\tau$ effect is common in daily life: When moving between two places, we have an experience of a smaller distance by airplane than by bus. The present results indicate that what is experienced in traveling a long distance is reproduced in the perception of motion over small ranges in the laboratory.
It is possible to consider the proprioceptive $\mathrm{S}$ effect, in that when the objective velocity at which the body rotates is constant, perceived velocity of self-motion increases as the objective range increases. The proprioceptive $\mathrm{S}$ effect is similar to the visual S effect (Abbe, 1936) or the $\kappa$ (kappa) effect (Cohen, Hansel, \& Sylvester, 1953), which means that when two stationary light points are successively presented with a constant time lag, the perceived time interval of the stimulus onset asynchrony increases as the objective distance between the points increases. The proprioceptive S effect, however, was not found in Experiment 1 and was limited to the $9.6 \% \mathrm{sec}$ velocity (i.e., the velocity $\times$ range interaction) or to the tilted roll (i.e., the roll $\times$ range interaction) in Experiment 2.

\section{Effects of Body Tilt}

In Experiment 1, the self-motion through tilted roll enlarged perceived range and perceived duration but reduced perceived velocity. In Experiment 2, the selfmotion through the tilted roll reduced perceived velocity for the $80^{\circ}$ range (i.e., the roll $\times$ range interaction). Why did the tilting of the body sometimes enlarge perceived range and perceived duration and reduce perceived velocity? Although other interpretations are possible, this may be due mainly to muscular efforts that are needed to support the tilted body. Proffitt, Stefanucci, Banton, and Epstein (2003) and Stefanucci, Proffitt, Banton, and Epstein (2005) have demonstrated that when walking effort is increased, we perceive distances to be farther and hills to be steeper. It seems that large muscular efforts affect not only perceived range (i.e., distance), but also perceived duration and perceived velocity. We know that when bringing a heavy object with us, we walk slowly, taking much time to arrive at a goal. The effects of body tilt found in this study agree with our actions and experiences in daily life.

\section{Effects of the Visual Vertical}

The effects of the visual vertical are twofold in this study. First, judgments of range and velocity in the exposure condition were more stable than those in the occlusion condition. This result supports the finding of Nemire and Cohen (1993), who showed that body orientation in the median plane is most stable when both visual and somatosensory inputs are available.

We also found several conditions in which the visual vertical affected the mean perceived range and perceived velocity of self-motion. First, the mean perceived range of self-motion through the vertical roll was more reduced in the exposure condition than in the occlusion condition. Second, the mean perceived velocity of self-motion over a small range through the vertical roll was smaller in the exposure condition than in the occlusion condition. These findings, taken together, suggest that when the visual vertical was viewed with a usual posture in which the longitudinal axis of the body moves within a small range around the gravitational vertical, the mean perceived range and perceived velocity were reduced. This supports the finding of Higashiyama and Koga (2002), who indicated that perceived velocity of self-motion is smaller when a participant is exposed to a stationary visual pattern than when 
he or she is prevented from seeing it. We are not aware that the head or trunk is constantly moving even if we make an effort to stabilize it. This unnoticed self-motion is understandable if the visual information is assumed to suppress activity of the vestibular system (Brandt, Bartenstein, Janek, \& Dieterich, 1998).

To summarize, perceived range and perceived velocity of self-motion are determined by multiple sensory inputs, including vestibular and somatosensory inputs. The gravity that acts on the body rotating in roll changes the vestibular and somatosensory inputs continuously and simultaneously. Owing to these sensory inputs, judgments of range are very accurate, but nevertheless, judgments of velocity are compressive. We think that the judgments of velocity are generated by a combination of an accurate perceived range and a compressive perceived duration.

Not only the vestibular and somatosensory, but also other sensory systems contribute to perceived range and perceived velocity of self-motion. There are three findings worth noting as the effects of the other sensory systems. The first is the proprioceptive $\tau$ effect: Perceived range of self-motion is affected by the velocity of selfmotion or by the time interval that is needed to move over the range. The next is that self-motion through the tilted roll enlarges perceived range and perceived duration but reduces perceived velocity. We attribute this effect to muscular efforts to support the tilted body. The last is that in self-motion over a small range across the gravitational vertical, perceived range and perceived velocity are reduced under an exposure to the visual vertical. The exposure to the visual vertical while the body moves also reduces the variability of the judgments of range and velocity. These effects are clearly due to visual inputs. We trust that these findings will be useful in resolving the complicated network of perceptual processing involved in passive self-motion.

\section{AUTHOR NOTE}

This study was partially supported by the Ritsumeikan Research fund and by Grant-in-Aid for Scientific Research, Basic Research (C) 20530672, from the Ministry of Education, Science, Sport, and Culture in Japan. We are grateful to Mark Hollins (University of North Carolina at Chapel Hill) for his critical reading of an earlier version of the manuscript. Correspondence should be addressed to K. Koga, EcoTopia Science Institute, Nagoya University, Chikusa-ku, Nagoya 464-8603, Japan (e-mail: koga@esi.nagoya-u.ac.jp), or to A. Higashiyama, Department of Psychology, Ritsumeikan University, Kita-ku, Kyoto 603-8577, Japan (e-mail: achan@1t.ritsumei.ac.jp).

\section{Note-Accepted by the previous editorial team, when Thomas H. Carr was Editor.}

\section{REFERENCES}

Aвbe, M. (1936). The temporal effect upon perception of space. Japanese Journal of Experimental Psychology, 3, 1-50.

Algom, D., \& Cohen-Raz, L. (1984). Visual velocity input-output functions: The integration of distance and duration onto subjective velocity. Journal of Experimental Psychology: Human Perception \& Performance, 10, 486-501.

Allison, R. S., Howard, I. P., \& Zacher, J. E. (1999). Effect of field size, head motion, and rotational velocity on roll vection and illusory self-tilt in a tumbling room. Perception, 26, 299-306.

BENSON, A. J. (1990). Sensory functions and limitations of the vestibular system. In R. Warren \& A. Wertheim (Eds.), Perception and control of self-motion (pp. 145-170). Hillsdale, NJ: Erlbaum.

Benson, A. J., Spencer, M. B., \& Stott, J. R. R. (1986). Thresholds for the detection of the direction of whole-body, linear movement in the horizontal plane. Aviation, Space, \& Environmental Medicine, 57, 1088-1096.

Brandt, T., Bartenstein, P., Janek, A., \& Dieterich, M. (1998). Reciprocal inhibitory visual-vestibular interaction: Visual motion stimulation deactivates the parieto-insular vestibular cortex. Brain, 121, 1749-1758.

Bronstein, A. M. (1999). The interaction of otolith and proprioceptive information in the perception of verticality: The effects of labyrinthine and CNS disease. In B. Cohen \& B. J. M. Herr (Eds.), Otolith function in spatial orientation and movement (Annals of the New York Academy of Sciences, Vol. 871, pp. 324-333). New York: New York Academy of Sciences.

Brown, J. H. (1966). Magnitude estimation of angular velocity during passive rotation. Journal of Experimental Psychology, 72, 169-172.

BROWN, S. W. (1997). Attentional resources in timing: Interference effects in concurrent temporal and nontemporal working memory tasks. Perception \& Psychophysics, 59, 1118-1140.

Clark, B. (1967). Thresholds for the perception of angular acceleration in man. Aerospace Medicine, 38, 443-450.

Clark, B., \& Stewart, J. D. (1968a). Comparison of three methods to determine thresholds for perception of angular acceleration. American Journal of Psychology, 81, 207-216.

Clark, B., \& Stewart, J. D. (1968b). Magnitude estimates of rotational velocity during and following prolonged increasing, constant, and zero angular acceleration. Journal of Experimental Psychology, 78, 329-339.

Clark, F. J., \& Horch, K. W. (1986). Kinesthesis. In K. R. Boff, L. Kaufman, \& J. P. Thomas (Eds.), Handbook of perception and human performance: Vol. 1. Sensory processes and perception (pp. 13.1-13.63). New York: Wiley.

Cohen, J., Hansel, C. E. M., \& Sylvester, J. D. (1953). A new phenomenon in time judgment. Nature, 172, 901-903.

Dichgan, J., Held, R., Young, L. R., \& Brandt, T. (1972). Moving visual scenes influence the apparent direction of gravity. Science, 178, 1217-1218.

EISLER, H. (1976). Experiments on subjective duration 1868-1975: A collection of power function exponents. Psychological Bulletin, 83, 1154-1171.

Helson, H., \& King, S. M. (1931). The tau effect: An example of psychological relativity. Journal of Experimental Psychology, 14, 202-217.

Hicks, R. E., Miller, G. W., Gaes, G., \& Bierman, K. (1977). Concurrent processing demands and the experience of time-in-passing. American Journal of Psychology, 90, 431-446.

Hicks, R. E., Miller, G. W., \& Kinsbourne, M. (1976). Prospective and retrospective judgments of time as a function of amount of information proceeded. American Journal of Psychology, 89, 719-730.

Higashiyama, A., \& Koga, K. (1998). Apparent body tilt and postural aftereffect. Perception \& Psychophysics, 60, 331-347.

Higashiyama, A., \& Koga, K. (2002). Integration of visual and vestibulo-tactile inputs affecting apparent self-motion around the line of sight. Perception \& Psychophysics, 64, 981-995.

Higashiyama, A., \& Koga, K. (in press). Perceived self-tilt in dynamic visual stimuli: Evidence for suppression by vestibulo-tactile inputs. Perception.

Hollins, M., \& Goble, A. K. (1988). Perception of the length of voluntary movements. Somatosensory Research, 5, 335-348.

Howard, I. (1982). Human visual orientation. Chichester, U.K.: Wiley. LACKNER, J. R. (1992). Spatial orientation in weightless environments. Perception, 21, 803-812.

LACKNER, J. R., \& DiZIo, P. (2005). Vestibular, proprioceptive, and haptic contributions to spatial orientation. Annual Review of Psychology, 56, $115-147$

LACKNER, J. R., \& Graybiel, A. (1979). Parabolic flight: Loss of sense of orientation. Science, 206, 1105-1108.

Lackner, J. R., \& Graybiel, A. (1983). Perceived orientation in freefall dependents on visual, postural, and architectural factors. Aviation, Space, \& Environmental Medicine, 54, 47-51. 
LeChNER-STEINLEITNER, S. (1978). Interaction of labyrinthine and somatoreceptor inputs as determinants of the subjective vertical. Psychological Research, 40, 65-76.

LockHART, J. M. (1967). Ambient temperature and time estimation. Journal of Experimental Psychology, 73, 286-291.

LonG, G. M., \& BeATON, R. J. (1980). The contribution of visual persistence to the perceived duration of brief targets. Perception \& Psychophysics, 28, 422-430.

NeMIRE, K., \& CoHEN, M. M. (1993). Visual and somesthetic influences on postural orientation in the median plane. Perception \& Psychophysics, 53, 106-116.

ONO, F., \& KaWAHARA, J. (2007). The subjective size of visual stimuli affects the perceived duration of their presentation. Perception \& Psychophysics, 69, 952-957.

Parker, D. E., Gulledge, W. L., Tubbs, R. L., \& Littlefield, V. M. (1978). A temporary threshold shift for self-motion detection following sustained, oscillating linear acceleration. Perception \& Psychophysics, 23, 461-467.

Parker, D. E., Wood, D. L., Gulledge, W. L., \& Goodrich, R. L. (1979). Self-motion magnitude estimation during linear oscillation: Changes with head orientation and following fatigue. Aviation, Space, \& Environmental Medicine, 50, 1112-1121.

Proffitt, D. R., Stefanucci, J., Banton, T., \& Epstein, W. (2003). The role of effort in perceiving distance. Psychological Science, 14, 106-112.

RaCHLIN, H. C. (1966). Scaling subjective velocity, distance, and duration. Perception \& Psychophysics, 1, 77-82.
Reason, J. T., Mayes, A. R., \& Dewhurst, D. (1982). Evidence for boundary effect in roll vection. Perception \& Psychophysics, 31, 139-144.

Stefanucci, J. K., Proffitt, D. R., Banton, T., \& Epstein, W. (2005). Distances appear different on hills. Perception \& Psychophysics, 67, 1052-1060.

Treisman, M., Cook, N., Naish, P. L. H., \& MacCrone, J. K. (1994). The internal clock: Electroencephalographic evidence for oscillatory processes underlying time perception. Quarterly Journal of Experimental Psychology, 47A, 241-289.

van Beuzekom, A. D., Medendorp, W. P., \& van Gisbergen, J. A. M. (2001). The subjective vertical and the sense of self orientation during active body tilt. Vision Research, 41, 3229-3342.

van Beuzekom, A. D., \& van Gisbergen, J. A. M. (2000). Properties of the internal representation of gravity inferred from spatial-direction and body-tilt estimates. Journal of Neurophysiology, 84, 11-27.

WitkIN, H. A. (1949). Perception of body position and of the position of the visual field. Psychological Monographs, 63 (7, Whole No. 302).

YARDLEY, L. (1990). Contribution of somatosensory information to perception of the visual vertical with body tilt and rotating visual field. Perception \& Psychophysics, 48, 131-134.

Young, L. R., Oman, C. M., \& Dichgans, J. M. (1975). Influence of head orientation on visually induced pitch and roll sensation. Aviation, Space, \& Environmental Medicine, 46, 264-268.

(Manuscript received February 5, 2007; revision accepted for publication July 14, 2008.) 